\title{
Calcipotriol/Betamethasone Dipropionate Foam Inhibits Th17 Cytokine Secretion and Improves Epidermal Barrier Markers in a Human Th17 Skin Inflammation Model
}

Paola Lovato · Li Jiang · Josephine Hebsgaard · David A. Ewald •

Hanne Norsgaard (D)

Received: November 27, 2020 / Accepted: December 24, 2020 / Published online: January 17, 2021

(C) The Author(s) 2021

\begin{abstract}
Introduction: T-helper 17 (Th17) cytokines play a key role in the pathophysiology of psoriasis by driving inflammatory responses that lead to epidermal alterations. Markers of epidermal differentiation, including the proteins loricrin (LOR), filaggrin (FLG) and involucrin (IVL), are dysregulated in psoriatic skin. The fixed-dose combination of calcipotriol/betamethasone dipropionate (Cal/BD) foam and clobetasol propionate (CP) are widely used, effective topical treatments for psoriasis. In this study, we investigated the effects of $\mathrm{Cal} / \mathrm{BD}$ foam and CP cream on Th17 cytokine secretion and epidermal differentiation using a human Th17 skin inflammation model (InflammaSkin ${ }^{\circledR}$ ).

Methods: The fixed-dose combination Cal/BD foam and the CP cream were applied once and twice daily, respectively, onto the air-exposed epidermal surface of InflammaSkin cultures for 7 days. Th17 cytokine levels were measured in culture supernatants, and gene expression analysis and immunohistochemical staining for LOR, FLG and IVL were performed on the skin samples.
\end{abstract}

P. Lovato · L. Jiang · J. Hebsgaard · D. A. Ewald .

H. Norsgaard $(\square)$

Skin Research, LEO Pharma A/S, Ballerup, Denmark

e-mail: hnddk@leo-pharma.com
Results: Topical treatment with Cal/BD foam almost completely inhibited Th17 cytokine secretion and upregulated LOR and IVL expression, but not FLG expression, at the mRNA and protein levels. Topical treatment with $\mathrm{CP}$ cream significantly reduced Th17 cytokine levels, but to a lesser extent than Cal/BD foam, and did not improve expression of any of the epidermal differentiation markers.

Conclusion: Compared with CP treatment, the fixed-dose combination Cal/BD foam showed a greater suppression of Th17 cytokine secretion and improved epidermal differentiation, resulting in an overall higher degree of improvement of the skin. These results support our understanding of the mechanisms behind the clinical efficacy observed for Cal/BD foam and of its use for long-term proactive treatment of psoriasis vulgaris.

Keywords: Betamethasone dipropionate; Calcipotriol; Clobetasol propionate; Psoriasis; Skin barrier; Th17 cytokines 


\section{Key Summary Points}

\section{Why carry out this study?}

Topical treatment is used by the majority of patients with psoriasis vulgaris and should be suitable for long-term management to maintain skin clearance of affected areas with recurring flares resulting from perturbation of the skin barrier and from reactivation of resident inflammatory cells.

The aim of this study was to investigate the effect of two widely used topical treatments, calcipotriol/betamethasone dipropionate (Cal/BD) foam and clobetasol propionate (CP) cream, on T-helper 17 (Th17) cytokine secretion and epidermal differentiation using a human Th17 skin inflammation model (InflammaSkin ${ }^{\circledR}$ ).

\section{What was learned from the study?}

A higher degree of improvement of the skin, with greater suppression of Th17 cytokines and induction of markers of epidermal differentiation, was seen after treatment with $\mathrm{Cal} / \mathrm{BD}$ foam compared with CP cream.

The results highlight the additive and/or complementary modes-of-action of calcipotriol and betamethasone dipropionate combination and supports our understanding of the mechanisms underlying its clinical efficacy.

The modulatory effects of calcipotriol on inflammatory cytokines and on epidermal differentiation together with the broad anti-inflammatory activity of betamethasone dipropionate effectively suppress inflammation and may prevent onset of new psoriatic lesions, supporting recent clinical data on the use of $\mathrm{Cal} / \mathrm{BD}$ foam for long-term proactive treatment of psoriasis vulgaris.

\section{DIGITAL FEATURES}

This article is published with digital features, including a summary slide, to facilitate understanding of the article. To view digital features for this article go to https://doi.org/10.6084/ m9.figshare.13482615.

\section{INTRODUCTION}

Psoriasis vulgaris is a chronic inflammatory, immune-mediated disease characterised by well-delineated, erythematous, scaly, thickened plaques. The clinical features are due to enhanced vascularisation, immune cell infiltration and epidermal hyperplasia with the formation of rete ridges, hypogranulosis and parakeratosis. T-helper 17 (Th17) cells play a key role in the pathophysiology of psoriasis by producing cytokines (interleukin [IL]-17A, IL$17 \mathrm{AF}$, IL-17F and IL-22) that act on keratinocytes to induce further pro-inflammatory mediators, such as IL-17C, which together cause activation, hyperproliferation and premature differentiation of keratinocytes [1]. The incomplete cornification of keratinocytes in psoriasis is reflected by the dysregulated expression and localisation of epidermal differentiation markers, such as the proteins loricrin (LOR), filaggrin (FLG) and involucrin (IVL), which are important for maintaining a normal skin barrier $[2,3]$.

The majority of patients with psoriasis vulgaris have localised, limited skin manifestations $(<5 \%$ body surface area involvement) that are suitable for topical treatment [4]. The fixed-dose combination calcipotriol/betamethasone dipropionate (Cal/BD) foam and the potent corticosteroid clobetasol propionate (CP) are widely used, effective topical psoriasis treatments $[4,5]$. However, even if successful treatment to clinical resolution is achieved, the chronic nature of the disease, characterised with periods of remission and recurring flares in the same areas due to remaining inflammatory cells [6], requires topical treatments appropriate for long-term management to maintain skin clearance. Recent data show that long-term, twiceweekly proactive treatment with $\mathrm{Cal} / \mathrm{BD}$ foam for psoriasis vulgaris can increase both the time 
to first recurrence and time in remission over 52 weeks with an unchanged safety profile as compared to reactive treatment with $\mathrm{Cal} / \mathrm{BD}$ foam over the same period [7].

Calcipotriol, a vitamin D receptor (VDR) agonist, has been shown to inhibit Th17 and augment Th2 and regulatory $\mathrm{T}$-cell responses $[8,9]$. In contrast, corticosteroids have a broad anti-inflammatory and immunosuppressive effect [10]. Furthermore, corticosteroids and VDR agonists have opposing effects on the skin barrier [11-13]. Studies in healthy volunteers and mice show that short-term topical corticosteroid treatment compromises skin barrier homeostasis and stratum corneum integrity by impeding lipid synthesis [11]. Long-term topical corticosteroid use results in suppression of epidermal differentiation and depletion of stratum corneum intercellular lipids, which are important for maintaining barrier function [12]. Conversely, VDR agonists restore and maintain skin barrier function by promoting epidermal differentiation and lipid synthesis [13].

Most data support greater efficacy or antiinflammatory activity with $\mathrm{Cal} / \mathrm{BD}$ combination therapy versus corresponding monotherapies $[8,14,15]$. To elucidate the mechanisms underlying these clinical findings, the aim of this study was to investigate effects of the fixeddose combination Cal (0.005\%)/BD (0.064\%) foam compared with CP $0.05 \%$ cream on Th17 cytokine secretion and epidermal differentiation using a recently developed human Th17 skin inflammation model (InflammaSkin ${ }^{\circledR}$; Genoskin,Toulouse, France), which reproduces inflammatory features of psoriasis and can be used to investigate pharmacological responses to topically applied treatments [16].

\section{METHODS}

\section{InflammaSkin Model and Topical Treatment}

Topical treatment of Cal/BD foam and CP cream in the InflammaSkin model was performed at Genoskin (Toulouse, France). Anonymised human skin samples were collected from four healthy donors who had undergone abdominoplasty procedures and had given their written informed consent. Full ethical approval for the study protocol was obtained from the French ethical research committee (Comité de Protection des Personnes), and authorisation was given by the French Ministry of Research. The study was conducted according to the Declaration of Helsinki. Full-thickness skin punch biopsies (diameter $15 \mathrm{~mm}$ ) were embedded in a matrix, with the epidermal surface exposed to the air (NativeSkin ${ }^{\circledR}$ model; Genoskin). For the InflammaSkin model, biopsies were injected with anti-CD3/CD28 antibodies plus IL-2 to induce in situ activation of skin resident $\mathrm{T}$ cells before insertion into the matrix. Thereafter, IL-1 $\beta$, IL-23 and transforming growth factor- $\beta$ were added during ex vivo culture to promote a Th17 phenotype [16].

Topical treatment of Cal/BD foam and CP cream was applied once and twice daily (as per the labels), respectively, onto the air-exposed epidermal surface at initiation of ex vivo culture for a total of 7 days. Untreated InflammaSkin cultures were used as positive controls; untreated healthy skin cultures (NativeSkin ${ }^{\circledR}$ ) were used as negative controls. Duplicates were generated from all donors for each treatment group. All samples were shipped to LEO Pharma A/S (Ballerup, Denmark) for analysis.

\section{Cytokine Analysis}

Cytokine levels in culture supernatants were assessed using Meso Scale Discovery assay kits (Meso Scale Discovery Rockville, MD, USA) for IL-17AF (K151VYK-1), IL-17A and IL-17C (K15067L-1), and R\&D enzyme-linked immunosorbent assay kits (R\&D Systems, Minneapolis, MN, USAUK) for IL-17F (DY1335B) and IL-22 (D2200), following each manufacturer's instructions. Cytokine concentrations were calculated by comparison to the standard curve, which was generated in the same biological matrix as the samples. The results were expressed as the percentage of untreated controls for each experiment, and statistical analysis of treatment effects was performed using one-way analysis of variance followed by Tukey's multiple comparison test based on a 95\% confidence interval. 


\section{Gene Expression Analysis}

Gene expression analysis of $L O R, F L G$ and IVL was performed on NativeSkin and InflammaSkin samples by quantitative real-time PCR (qPCR) using Taqman ${ }^{\circledR}$ gene expression assays (LOR-Hs01894962_s1, FLG-Hs00856927_g1, IVL-Hs00846307_s1, HPRT1- Hs99999909_m1, PGK1- Hs99999906_m1, PPIA-Hs99999904_m1, GAPDH-Hs99999905_m1), of which HPRT1, PGK1, PPIA and GAPDH were used as reference genes (Applied Biosystems, Thermo Fisher Scientific, Foster City, CA USA). Normalised differential expression was calculated between the respective groups and the InflammaSkin-positive control group using the moderated $t$ test of the 'R/limma' software framework.

\section{Immunohistochemistry}

Staining of LOR, FLG and IVL was performed on formalin-fixed and paraffin-embedded sections from NativeSkin and InflammaSkin samples. As reference, staining was also performed on sections of anonymised samples from normal skin and paired lesional and non-lesional psoriasis skin. Staining was performed using anti-LOR (0.5 $\mathrm{gg} / \mathrm{mL}$; ab85679; Abcam, Cambridge, UK), anti-FLG $(0.8 \mu \mathrm{g} / \mathrm{mL}$; LS-B13455; Nordic BioSite ApS, Copenhagen, Denmark) and anti-IVL $(0.1 \mu \mathrm{g} / \mathrm{mL}$; PA5-32,454; Thermo Fisher Scientific, Waltham, MA, USA) rabbit antibodies. Antibodies were detected with the Bond Refine RED kit (DS9390; Leica Biosystems, Wetzlar, Germany) with BrightVision poly AP-Anti-rabbit IgG (KDPVR110AP; ImmunoLogic, Duiven, The Netherlands) as secondary antibody. Nuclei were stained blue with haematoxylin.

\section{RESULTS}

To investigate the effects of Cal/BD foam and CP cream on Th17 cytokine secretion and epidermal differentiation, we used the recently developed InflammaSkin model. Treatments were topically applied at initiation of ex vivo culture for a total of 7 days.

Th17 cytokines (IL-17A, IL-17AF, IL-17F, IL22) were measured in the supernatants of the cultures; they were undetectable in untreated healthy skin cultures (NativeSkin) but induced in InflammaSkin cultures (Fig. 1), as previously described [16]. Secretion of IL-17C was detected in the NativeSkin control and was significantly increased in the InflammaSkin cultures (Fig. 1). Once-daily treatment with Cal/BD foam significantly reduced cytokine levels, with almost complete suppression of all Th17 cytokines tested (percentage inhibition and significance $[P]$ vs. untreated InflammaSkin cultures: IL-17A 98.4\%, $P<0.01$; IL-17AF 96.6\%, $P<0.001$; IL17C 53.1\%, $P<0.001$; IL-17F 95.9\%, $P<0.001$; IL-22 91.2\%, $P<0.01$ ) (Fig. 1). Twice-daily treatment with CP cream also reduced cytokine levels but overall to a lesser extent than Cal/BD foam (percentage inhibition and significance $(P)$ vs untreated InflammaSkin cultures: IL-17A 89.9\%, $P<0.01$; IL-17AF 68.6\%, not significant [ns]; IL-17C 53.5\%, ns; IL-17F 86.2\%, $P<0.01$; IL-22 88.3\%, $P<0.01)$. A direct comparison of the effects of Cal/BD foam versus CP cream did not result in statistically significant differences.

After ex vivo culture for 7 days, gene expression levels of $L O R, F L G$ and $I V L$ were analysed and found to be downregulated in InflammaSkin samples relative to NativeSkin samples, with $L O R$ and FLG being downregulated to the greatest extent (Fig. 2). Treatment with Cal/BD foam significantly upregulated gene expression of $L O R$ and IVL $(P<0.01$ for both vs. positive control) but not $F L G$, while no improvement was observed on any of the epidermal differentiation markers after CP treatment (Fig. 2). Interestingly, the treatment effect by $\mathrm{Cal} / \mathrm{BD}$ was significantly superior to that of CP (Fig. 2; $P<0.01-0.001$ ).

We then investigated protein expression of the epidermal differentiation markers by Immunohistochemical staining. For reference, samples of normal skin from four healthy donors and paired lesional and non-lesional skin biopsies from eight patients with untreated, moderate-to-severe psoriasis were stained for LOR, FLG and IVL. Normal and nonlesional psoriasis skin showed continuous LOR protein coverage in the stratum granulosum and stratum corneum whereas expression was patchy and extended into stratum spinosum in lesional psoriasis skin (Fig. 3a). The localisation 

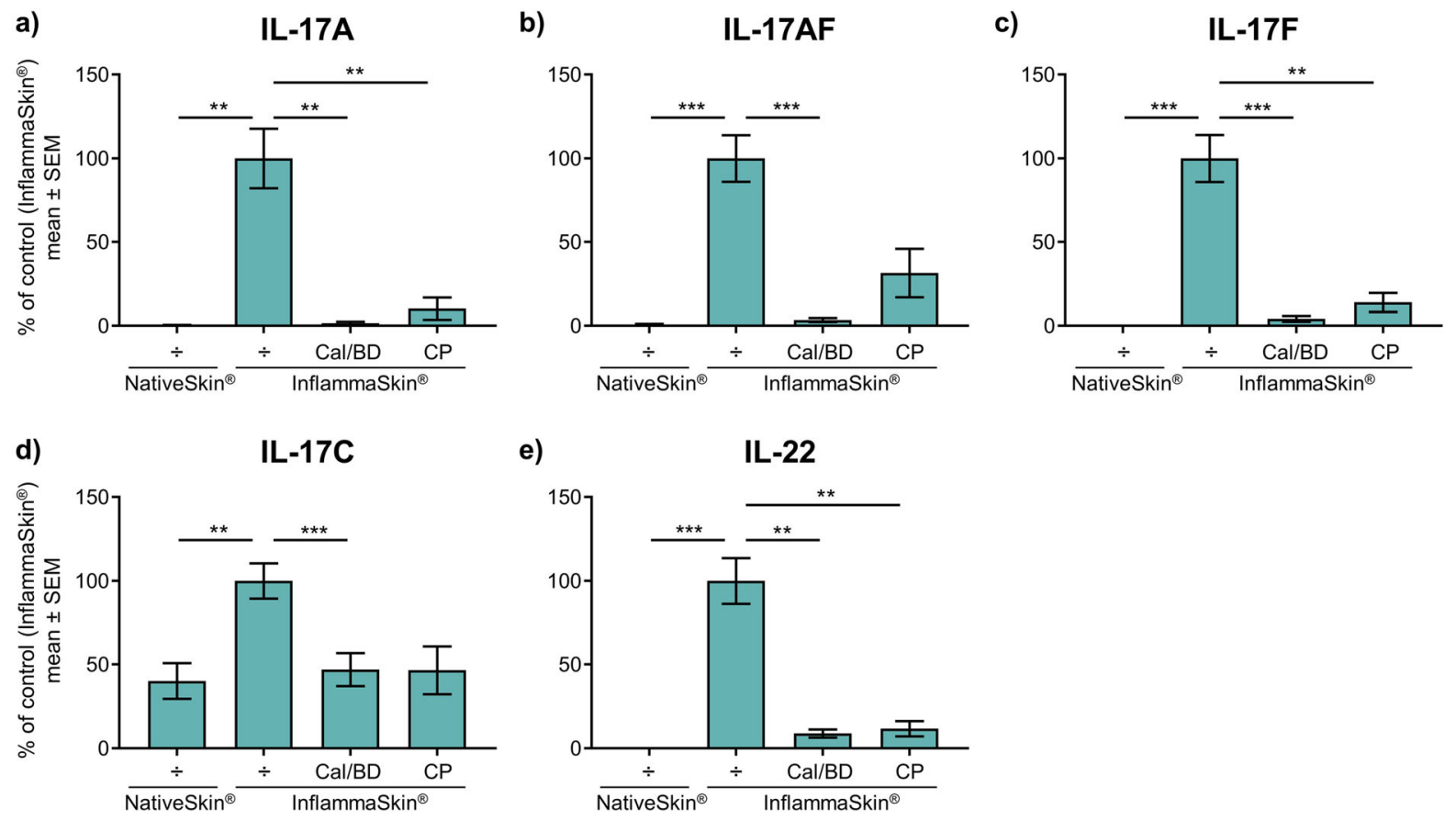

Fig. 1 Effect of Cal/BD foam and CP cream on T-helper 17 and IL-17C cytokine levels. Secreted cytokine levels of IL-17A (a), IL-17AF (b), IL-17F (c), IL-17C (d) and IL22 (e) in culture supernatants of treated and control InflammaSkin ${ }^{\circledR}$ and NativeSkin ${ }^{\circledR}$ models from four donors (each donor with duplicate samples per treatment group). Protein levels (mean \pm SEM) are calculated as percentage $(\%)$ of the InflammaSkin control. One-way

of FLG protein was similar to that of LOR in normal, lesional and non-lesional skin (Fig. 3a). Compared with LOR and FLG, the expression pattern of IVL protein was broader, extending into the stratum spinosum in normal skin. IVL was markedly upregulated in lesional and nonlesional psoriasis skin, covering the stratum granulosum, stratum spinosum and, to a lesser extent, stratum basale (Fig. 3a).

For the skin models, levels of LOR protein varied among the four donors but was present in all NativeSkin samples and was almost completely absent in InflammaSkin samples, in agreement with the downregulation seen at the gene expression level (Figs. 2b, 3b). Furthermore, protein expression of LOR was partially normalised by Cal/BD foam, with little/no effect of CP cream. The treatment effect on FLG gene expression could not be confirmed at the analysis of variance, followed by Tukey's multiple comparison test, was used to compare effects between treatment groups. Asterisks indicate significant difference at ${ }^{* *} P<0.01 ;{ }^{* * *} P<0.001 ; \div$ indicates no treatment. Cal Calcipotriol, Cal/BD fixed-dose combination of calcipotriol/betamethasone dipropionate, $C P$ clobetasol propionate, $I L$ interleukin, $S E M$ standard error of the mean

protein level due to high variation of FLG protein expression seen among both NativeSkin and InflammaSkin samples (data not shown). Protein expression of IVL was decreased in InflammaSkin compared with NativeSkin samples, as also seen at the gene expression level (Figs. 2c, 3c). The expression of IVL was partially reversed by Cal/BD foam, replicating NativeSkin expression pattern, whereas no effect was seen by CP cream.

\section{DISCUSSION}

Topical treatment with $\mathrm{Cal} / \mathrm{BD}$ foam in the InflammaSkin model almost completely inhibited Th17 cytokine secretion and upregulated LOR and IVL expression at the mRNA and protein level. CP cream significantly reduced Th17 


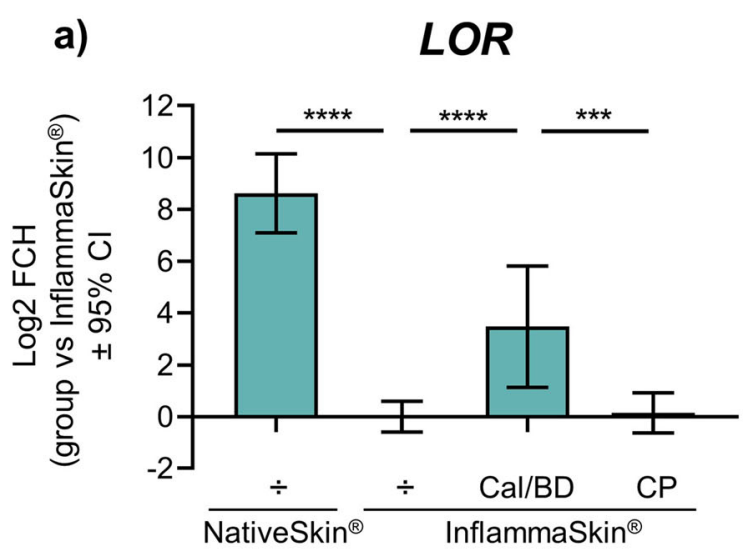

4Fig. 2 Effect of $\mathrm{Cal} / \mathrm{BD}$ foam and $\mathrm{CP}$ cream on the expression of genes associated with epidermal differentiation. Gene expression levels of $\operatorname{LOR}(\mathbf{a}), F L G(\mathbf{b})$ and $I V L$ (c) in skin samples of treated and control InflammaSkin and NativeSkin models from four donors (each donor with duplicate samples per treatment group). Normalised differential expression between the respective groups and the InflammaSkin control is shown as $\log _{2}$ fold change \pm 95\% CI. Statistical analysis was performed using the moderated $t$ test of the 'R/limma' framework. Asterisks indicate significant difference at ${ }^{*} P<0.05$; ${ }^{* *} P<0.01$; ${ }^{* * *} P<0.001 ;{ }^{* * * *} P<0.0001 ; \div$,indicates no treatment. $C I$ Confidence interval, $F C H$ fold change, $F L G$ filaggrin, $I V L$ involucrin, $L O R$ loricrin

b)

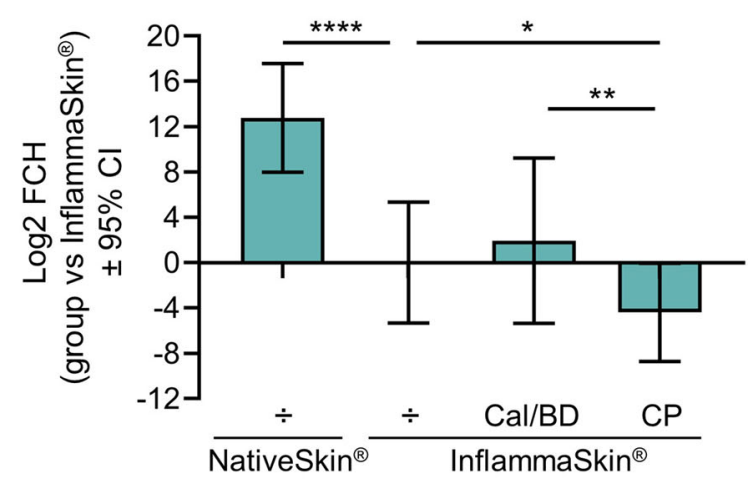

c)

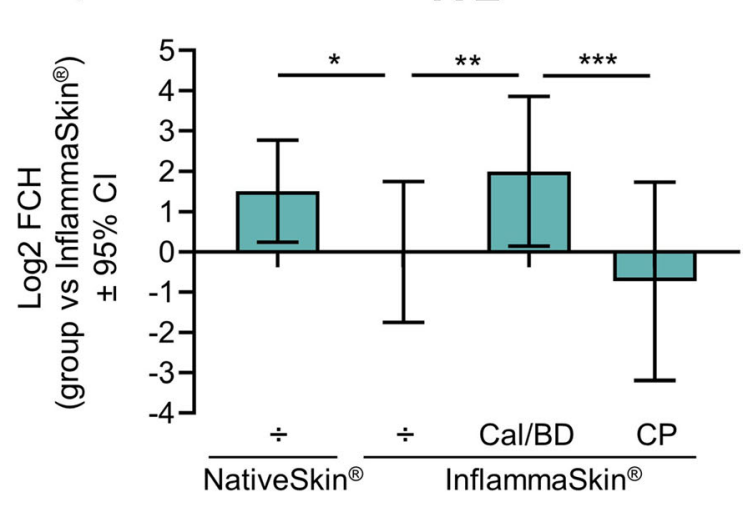

cytokine levels, but to a lesser extent than Cal/ $\mathrm{BD}$ foam, and did not improve LOR and IVL expression, highlighting the additive and/or complementary effects by calcipotriol on Th17 cytokine secretion and on epidermal differentiation, respectively, as previously reported $[8,9,13,17]$.

Whereas alterations of LOR and FLG expression in the NativeSkin versus InflammaSkin cultures overall reflected a similar change in expression pattern as seen in normal/non-lesional versus lesional psoriasis skin, this was not the case for IVL. Compared with NativeSkin samples, IVL expression was decreased in InflammaSkin samples, not mimicking the increased and extended expression of IVL that we found in psoriasis skin, as also reported in a previous study [3]. This discrepancy may partly be due to limited hyperplasia and lack of rete ridges in the InflammaSkin model. In addition, the presence of necrotic cells in the upper layers of the epidermis in the skin model may explain the lack of IVL superficial expression. Although the InflammaSkin model captures crosstalk between Th17 cells and keratinocytes and reproduces features of skin inflammation observed in psoriatic lesions, the model has its limitations and does not replicate the full complexity of psoriasis pathology.

The contrasting effect of corticosteroids and VDR agonists on skin barrier function [11-13] likely accounts for the improvements in epidermal differentiation markers observed with 


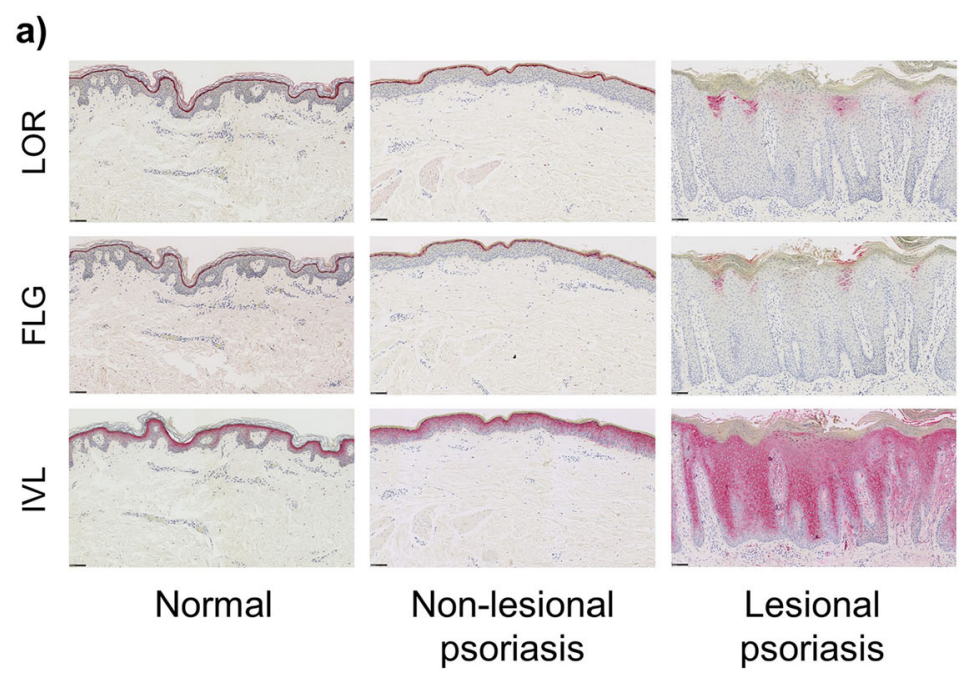

b)
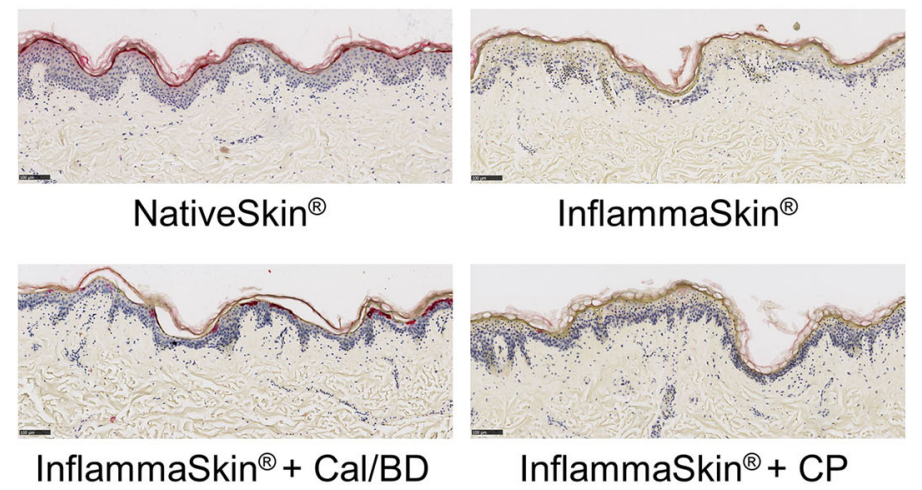

c)
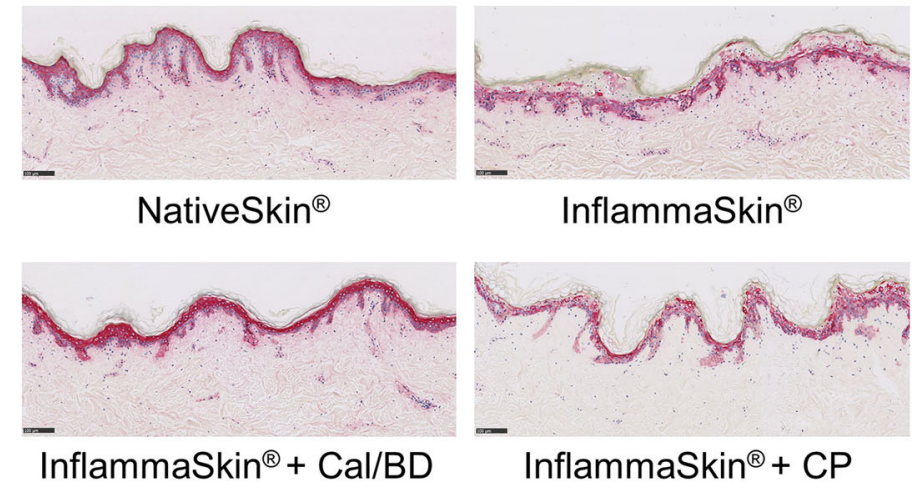

Fig. 3 Localisation of LOR, FLG and IVL proteins by immunohistochemical staining. a Localisation of LOR, FLG, and IVL in normal, non-lesional and lesional IVL (c) in treated and control InflammaSkin and NativeSkin models. A representative donor of four donors (each donor with duplicate samples per treatment group) is psoriasis skin samples. b, c Localisation of LOR (b) and shown. Scale bars: $100 \mu \mathrm{m}$ 
Cal/BD foam, which were not observed with CP cream. Perturbation of the epidermal barrier can initiate skin inflammation by inducing innate immune responses from keratinocytes and promoting the release of pro-inflammatory cytokines, such as IL-1 $\alpha$ [18], via the activation of transcription factors such as nuclear factor kappa light-chain-enhancer of activated B cells (NF-kB). The initiation of these early inflammatory processes leads to the activation of resident cells from the adaptive immune system, infiltration of circulating immune cells and, ultimately, to the beginning of a chronic skin inflammation. Accordingly, topical corticosteroid-induced skin barrier impairment may trigger inflammatory responses in a rebound effect via the increased activity of NF-kB, which is suppressed by the anti-inflammatory action of corticosteroids during treatment but appears after its cessation [19]. In contrast, VDR agonists have been shown to inhibit NF-kB activity [20] and consequently the secretion of IL-1 $\alpha$ and IL-8 by human keratinocytes [21].

Taken together, these findings support the complementary modes of action of Cal and BD. Due to the promoting effect on epidermal differentiation and lipid synthesis [13], as well as the immunomodulatory action of calcipotriol $[8,9,17], \mathrm{Cal} / \mathrm{BD}$ foam may prevent the onset of new psoriatic lesions.

A limitation of this study is that the results are based on findings from short-term treatment in an ex vivo human Th17 skin inflammation model, where the epidermal surface is exposed to a high humidity. However, the translational aspect of this model has been previously shown [16], supporting the findings of this work.

\section{CONCLUSION}

Our study using a recently developed human Th17 skin inflammation model showed that treatment with $\mathrm{Cal} / \mathrm{BD}$ foam resulted in an overall higher degree of improvement of the skin than CP cream, with greater impact on Th17 cytokines and epidermal differentiation.

These mechanistic findings support a better understanding of the molecular mechanisms underlying the clinical effects of the fixed-dose combination Cal/BD foam.

\section{ACKNOWLEDGMENTS}

Funding. Funding of this study, including the journal's Rapid Service Fee, was provided by LEO Pharma A/S.

Technical Assistance. The authors thank Claire Jardet, Genoskin, for running the InflammaSkin ${ }^{\circledR}$ model study, and Hanne Rosendal, Lili Rohde, Marianne Munk Petersen, Trine Gejsing and Liselotte B. Gurzulidis, all LEO Pharma A/S employees, for technical assistance.

Authorship. All named authors meet the International Committee of Medical Journal Editors (ICMJE) criteria for authorship for this article, take responsibility for the integrity of the work as a whole, and have given their approval for this version to be published.

Authorship Contributions. Hanne Norsgaard designed the study. All authors contributed to the data collection, analysis, interpretation and writing of the results. Paola Lovato and Hanne Norsgaard wrote the main draft of the manuscript.

Prior Presentation. This manuscript is based on work that has previously been presented at the American Academy of Dermatology Association (AAD) Annual Meeting 2019, Washington D.C., USA, March 01-05, 2019 (Poster 10086).

Disclosures. Paola Lovato, Li Jiang, Josephine Hebsgaard, David A. Ewald, and Hanne Norsgaard are employees of LEO Pharma A/S, which sponsored this study.

Compliance with Ethics Guidelines. The InflammaSkin ${ }^{\circledR}$ model was performed at Genoskin, France which collected anonymised human skin samples from healthy donors who had undergone abdominoplasty procedures and had given their written informed consent. Full 
ethical approval for the study protocol was obtained from the French ethical research committee (Comité de Protection des Personnes) and authorisation was given by the French Ministry of Research. The study was conducted according to the Declaration of Helsinki.

Data Availability. Data sharing is not applicable to this article as no datasets were generated or analysed during the current study.

Open Access. This article is licensed under a Creative Commons Attribution-NonCommercial 4.0 International License, which permits any non-commercial use, sharing, adaptation, distribution and reproduction in any medium or format, as long as you give appropriate credit to the original author(s) and the source, provide a link to the Creative Commons licence, and indicate if changes were made. The images or other third party material in this article are included in the article's Creative Commons licence, unless indicated otherwise in a credit line to the material. If material is not included in the article's Creative Commons licence and your intended use is not permitted by statutory regulation or exceeds the permitted use, you will need to obtain permission directly from the copyright holder. To view a copy of this licence, visit http://creativecommons.org/licenses/by$\mathrm{nc} / 4.0 /$.

\section{REFERENCES}

1. Martin DA, Towne JE, Kricorian G, et al. The emerging role of IL-17 in the pathogenesis of psoriasis: preclinical and clinical findings. J Invest Dermatol. 2013;133:17-26.

2. Kim BE, Howell MD, Guttman E, et al. TNF- $\alpha$ downregulates filaggrin and loricrin through c-Jun $\mathrm{N}$-terminal kinase: role for TNF- $\alpha$ antagonists to improve skin barrier. J Invest Dermatol. 2011;131: 1272-9.

3. Bernard BA, Reano A, Darmon YM, Thivolet J. Precocious appearance of involucrin and epidermal transglutaminase during differentiation of psoriatic skin. Br J Dermatol. 1986;114:279-83.
4. Menter A, Korman NJ, Elmets CA, et al. Guidelines of care for the management of psoriasis and psoriatic arthritis. Section 3. Guidelines of care for the management and treatment of psoriasis with topical therapies. J Am Acad Dermatol. 2009;60:643-59.

5. Paul C, Stein Gold L, Cambazard F, et al. Calcipotriol plus betamethasone dipropionate aerosol foam provides superior efficacy vs. gel in patients with psoriasis vulgaris: randomized, controlled PSO-ABLE study. J Eur Acad Dermatol Venereol. 2017;31:119-26.

6. Benezeder T, Wolf P. Resolution of plaque-type psoriasis: what is left behind (and reinitiates the disease). Semin Immunopathol. 2019;41:633-44.

7. Lebwohl M, Kircik L, Lacour J-P, et al. Twice-weekly topical calcipotriene/betamethasone dipropionate foam as proactive management of plaque psoriasis increases time in remission and is well tolerated over 52 weeks (PSO-LONG trial). J Am Acad Dermatol. 2020. https://doi.org/10.1016/j.jaad.2020. 09.037.

8. Fujiyama $\mathrm{T}$, Ito $\mathrm{T}$, Umayahara $\mathrm{T}$, et al. Topical application of a vitamin D3 analogue and corticosteroid to psoriasis plaques decreases skin infiltration of TH17 cells and their ex vivo expansion. J Allergy Clin Immunol. 2016;138:517-28.

9. Lovato P, Norsgaard H, Tokura Y, Røpke MA. Calcipotriol and betamethasone dipropionate exert additive inhibitory effects on the cytokine expression of inflammatory dendritic cell-Th17 cell axis in psoriasis. J Dermatol Sci. 2016;81:153-64.

10. Boumpas DT, Chrousos GP, Wilder RL, Cupps TR, Balow JE. Glucocorticoid therapy for immune-mediated diseases: basic and clinical correlates. Ann Intern Med. 1993;119:1198-208.

11. Kao JS, Fluhr JW, Man MQ, et al. Short-term glucocorticoid treatment compromises both permeability barrier homeostasis and stratum corneum integrity: inhibition of epidermal lipid synthesis accounts for functional abnormalities. J Invest Dermatol. 2003;120:456-64.

12. Sheu HM, Tai CL, Kuo KW, Yu HS, Chai CY. Modulation of epidermal terminal differentiation in patients after long-term topical corticosteroids. J Dermatol. 1991;18:454-64.

13. Hong SP, Oh Y, Jung M, et al. Topical calcitriol restores the impairment of epidermal permeability and antimicrobial barriers induced by corticosteroids. Br J Dermatol. 2010;162:1251-60.

14. Kaufmann R, Bibby AJ, Bissonnette R, et al. A New calcipotriol/betamethasone dipropionate formulation (Daivobet ${ }^{\mathrm{TM}}$ ) is an effective once-daily 
treatment for psoriasis vulgaris. Dermatology 2002;205:389-93.

15. Lebwohl M, Tyring S, Bukhalo $M$, et al. Fixed combination aerosol foam calcipotriene $0.005 \%$ (Cal) plus betamethasone dipropionate 0064\% (BD) is more efficacious than Cal or bd aerosol foam alone for psoriasis vulgaris: a randomized, doubleblind, multicenter, three-arm, phase 2 study. J Clin Aesthet Dermatol. 2016;9:34-41.

16. Jardet C, David A, Braun E, et al. Development and characterization of a human Th17-driven ex vivo skin inflammation model. Exp Dermatol. 2020;29(10):993-1003. https://doi.org/10.1111/ exd.14160.

17. Satake K, Amano T, Okamoto T. Calcipotriol and betamethasone dipropionate synergistically enhances the balance between regulatory and proinflammatory $\mathrm{T}$ cells in a murine psoriasis model. Sci Rep. 2019;9:16322.
18. Wood LC, Jackson SM, Elias PM, Grunfeld C, Feingold KR. Cutaneous barrier perturbation stimulates cytokine production in the epidermis of mice. J Clin Invest. 1992;90:482-7.

19. Lin TK, Wei $\mathrm{KJ}, \mathrm{Wu} \mathrm{CH}$, et al. Barrier abnormalities and keratinocyte-derived cytokine cascade after cessation of long-term topical glucocorticosteroid on hairless mouse skin. Dermatol Sin. 2015;33: 103-11.

20. Riis JL, Johansen C, Gesser B, et al. 1alpha, $25(\mathrm{OH})(2) \mathrm{D}(3)$ regulates NF-kappaB DNA binding activity in cultured normal human keratinocytes through an increase in IkappaBalpha expression. Arch Dermatol Res. 2004;296:195-202.

21. Zhang JZ, Maruyama K, Ono I, Iwatsuki K, Kaneko F. Regulatory effects of 1,25-dihydroxyvitamin D3 and a novel vitamin D3 analogue MC903 on secretion of interleukin-1 alpha (IL-1 $\alpha$ ) and IL- 8 by normal human keratinocytes and human squamous cell carcinoma call line (HSC-1). J Dermatol Sci. 1994;7:24-31. 